\title{
INFORMES
}

\section{La Declaración de Beijing sobre Población y Desarrollo}

\section{Preámbulo}

1. Nosotros, los PARLAMENTARIOS de diecinueve países de Asia, asistiendo a la primera Conferencia Asiática de Parlamentarios sobre Población y Desarrollo en Beijing, del 27 al 30 de octubre de 1981, y dirigiéndonos a las interrelaciones entre POBLACION y DESARROLLO:

CONSTATAMOS los esfuerzos hechos por los países de Asia en cuanto al desarrollo social y económico, y sus iniciativas respecto a la formulación c implementación de políticas de población y programas de planificación familiar durante las últimas dos décadas, éstos han contribuido de manera significativa al descenso de la tasa global de nacimiento.

RECONOCEMOS las relaciones inexplicables entre la población, los recursos y el medio ambiente para administrar los temas de la pobreza, el empleo y el desarrollo y en consecuencia reafirmar los objetivos y las metas de la DECLARACION DE COLOMBO SOBRE POBLACION Y DESARROLLO, y de la DECLARACION DE KUALA LUMPUR SOBRE RECURSOS, POBLACION Y DESARROLLO.

REITERAMOS la necesidad de los países de Asia de adoptar un enfoque que asegurará la integración apropiada de recursos y población en la formulación e implementación de sus políticas y programas de desarrollo.

AFIRMAMOS la necesidad de una cooperación mayor entre los países de Asia con respecto a sus esfuerzos de acelerar y avanzar el bienestar social y económico de sus pueblos, y a la vez respetando la soberanía nacional de cada país.

REAFIRMAMOS la necesidad de esfuerzos continuos para la realización y el establecimiento tempranos del Nuevo Orden Económico Internacional a fin de lograr una distribución justa y equitativa de los recursos mundiales, y, enfatizamos que estas metas se deben realizar por medio del fortalecimiento de los vínculos de solidaridad y cooperación entre los países de Asia.

2. ADMITIENDO la diversidad tanto de religiones y creencias como de culturas y tradiciones, y CONSCIENTES de las diferencias de condiciones socioeconómicas y políticas entre los países asiáticos, reconocemos a la vez que compartimos muchas INQUIETUDES en común:

- Que la población actual de Asia se estima sea 2.6 billones de personas, encubriendo casi el 60 por ciento de la población total mundial, y que para el año 2000 se anticipa un aumento de otro billón de personas.

- Que el 90 por ciento de los pobres del mundo viven en Asia, y, si las tendencias actuales continúan, esta situación se deteriorará aún más. Esta proporción tan grande de nuestra población padece de desnutrición, analfabetismo y enfermedad, y, por lo tanto, no goza plenamente ni de sus derechos humanos básicos ni de los beneficios del desarrollo. 
- Que aunque las mujeres constituyen casi el 50 por ciento de la población de los países de Asia, no se le ha concedido a este sector tan importante la oportunidad equitativa de participar, con eficacia, en el proceso de desarrollo ni de compartir sus beneficios.

- Que aunque casi el 60 por ciento de la población total de Asia, tenga menos de 25 años de edad, es decir, una estimación de 1.5 billones de personas en 1980 , no se le ha proporcionado a la juventud, segmento tan vigoroso de nuestros recursos humanos, las oportunidades adecuadas para desarrollar su potencialidad plena como ciudadanos y como líderes futuros.

- Que aunque muchos países de Asia estén dotados de recursos naturales abundantes logren un equilibrio a largo plazo entre población y recursos, con el fin de mejorar la calidad de la vida para nuestros pueblos, se limita en este momento por la carencia de los factores esenciales complementarios necesarios para su aprovechamiento eficaz y productivo.

- Que los resultados del desajuste entre la población y los recursos incluyen la desforestación, la erosión del subsuelo, y otros cambios ecológicos que conducen al deterioro del ambiente natural que, si no se frena, amenazarán a la existencia humana misma.

- Que la paz, la seguridad nacional y la estabilidad, son las precondiciones del desarrollo; y a menos que se les puedan asegurar en Asia, todos nuestros esfuerzos de mejorar la calidad de vida para nuestros pueblos fracasarán.

\section{Objetivos}

En vista de las inquietudes expresadas en el preámbulo, y reconociendo la necesidad de tratarlas más eficazmente, esta Conferencia, por lo tanto, se dirige hacia los siguientes objetivos:

1. PROMOVER la cooperación y la colaboración entre los Parlamentarios de los países de Asia a través de un mayor y más continuo intercambio de experiencias y conocimientos sobre los temas de población y desarrollo.

2. MEJORAR AUN MAS y enriquecer la calidad de vida de los pueblos asiáticos por medio del aprovechamiento y administración más eficaz de recursos, visto como la integración de la población, los recursos y el medio ambiente en el proceso de desarrollo.

3. LOGRAR la justicia social y el progreso económico a través de un mecanismo que con mayor eficacia acelerara el proceso, aprovechando un enfoque integrado y equilibrado de la población, los recursos y el desarrollo a nivel nacional, regional e internacional.

4. CONSOLIDAR los esfuerzos y fortalecer la cooperación entre los países de Asia a fin de lograr la realización y la creación del Nuevo Orden Económico Internacional. 


\section{Llamadas}

Esta conferencia, por lo tanto, llama a todos los parlamentarios de los paises de Asia a:

1. Fomentar la formación de grupos nacionales de Parlamentarios preocupados por los temas de población y desarrollo, y, a través de estos grupos:

- Incrementar la conciencia y promover el mayor entendimiento de las interrelaciones entre población y desarrollo entre los Parlamentarios.

- Iniciar, promover y apoyar programas de intercambio entre los Parlamentarios de los países de Asia y otros países del mundo, con el objetivo de desarrollar nuevas ideas y nuevos enfoques con respecto a estos temas.

- Promover el diálogo continuo entre los parlamentarios y los planificadores sociales, económicos y de población y el personal que tiene a su cargo implementará programas a nivel local, regional e internacional.

\section{Gobiemos y paises de Asia:}

2. No ignorar, durante la formulación de estrategias y programas para el desarrollo socioeconómico de sus pueblos, la abundancia de tradiciones intelectuales, filosóficas y culturales, sino aprovechar la riqueza de estos conocimientos y la base científica en que se sustenta para su aplicación directa a la planificación y los esfuerzos de implementación.

3. De acuerdo con las necesidades y las aspiraciones nacionales demostrar su voluntad política y dar mayor ímpetu a los programas de población existentes, otorgarles los recursos adecuados para satisfacer los requerimientos de estos programas, $y$. además adoptar politicas de población exhaustivas como parte integral de los planes nacionales de desarrollo.

4. Establecer entidades nacionales de coordinación donde no existan, para formular e implementar de manera eficaz, políticas y programas de población.

5. Emprender encuestas periódicas de población y examinar las tendencias de población y su impacto sobre la salud, la educación, el desarrollo agrícola e industrial, la vivienda y el medio ambiente.

6. Aumentar la asistencia financiera a los programas de planificación familiar y de población dentro de cada país, según sus necesidades.

7. Estimular y sostener la participación e involucrar a la comunidad en actividades de población y desarrollo a través del uso eficiente de los medios de comunicación y la movilización y utilización eficaces de los recursos comunitarios.

8. Promover y fortalecer la participación de los organismos voluntarios no gubernamentales en los programas de población y desarrollo a nivel local, nacional, regional e internacional, de acuerdo con un espíritu de esfuerzo y sociedad común. De acuerdo con este propósito, los gobiernos deberían fomentar que los organismos no gubernamentales voluntarios intensifiquen sus esfuerzos para acelerar el proceso de integración de población con el desarrollo, otorgándoles un lugar clave dentro del marco de las políticas nacionales.

9. Revisar los objetivos y las metas de los programas de población y desarrollo existentes, con el propósito de mejorar las posibilidades de alcanzar una tasa de crecimiento del uno por ciento para la región de Asia en el año 2000. 
10. Fortalecer y extender los programas socioeconómicos de desarrollo y asegurar que el desarrollo se dirija hacia la reduccion y la disminución de las disparidades sociales y económicas, y así acelerar la creación de una sociedad justa.

11. Asegurar que el proceso de desarrollo enfatice la autosuficiencia individual y comunitaria, creando entre el pueblo no sólo confianza en sí mismo, conciencia y responsabilidad social, sino también una percepción política más amplia orientadas hacia una visión popular de la planificación e implementación del desarrollo.

12. Planificar una estrategia acertada para la conservación de los recursos naturales y su utilización eficaz, a la vez que un reconocimiento de la necesidad de proteger el medio ambiente.

13. Ampliar las oportunidades para que las mujeres participen equitativamente en todos los niveles, aspectos políticos, económicos, sociales y culturales del desarrollo, tomando nota especial del Programa de Acción de las Naciones Unidas para la segunda mitad de la Década de la Mujer en los campos de Salud, Educación y Trabajo, adoptado en la Conferencia de Copenhague en julio de 1980.

14. Establecer e implementar leyes sobre los derechos familiares, cuando sean necesarias, para asegurar los derechos amplios y equitativos de hombres y mujeres, y elevar el nivel educativo de las mujeres para posibilitar un incremento de sus responsabilidades y derechos sociales, sobre todo en cuanto a su rol de madres.

15. Iniciar, promover y utilizar estudios e investigaciones para lograr un mejor entendimiento de los problemas de la juventud, para así introducir programas destinados a aprovechar este recurso humano tan vasto de acuerdo con los mejores intereses de su desarrollo futuro.

16. Promover la educación y capacitación vocacional básica, para garantizar una educación continua, integrar la educación sobre población, con los sistemas educativos formal e informal, como una preparación para que la juventud se realice como ciudadanos responsables y participe en los programas de desarrollo y población.

17. Reenfatizar su importancia e infundir la necesidad de lograr un equilibrio entre el desarrollo material y los valores espirituales, sobre todo entre los jóvenes, para así lograr un mayor beneficio en el desarrollo.

18. Asignar mayor atención a la incidencia creciente de la drogadicción (dependencia de narcóticos) y a las enfermedades venéreas.

19. Luchar por la realización temprana de las metas y los objetivos del Nuevo Orden Económico Internacional intensificando esfuerzos de cooperación en las áreas de interés mutuo.

20. Emprender medidas diseñadas para incrementar el comercio y la cooperación interasiática, para así aprovechar al máximo algún complemento económico, la disponibilidad de los recursos naturales y los mercados potenciales que existen entre los países de Asia.

21. Incrementar el diálogo significativo entre los países desarrollados y los que están en vías de desarrollo para mejorar las relaciones comerciales y efectuar una división más equitativa de recursos, tecnología y conocimientos. 


\section{Todos los gobiernos:}

22. Incrementar los subsidios generales o la asistencia internacional a los programas de población de la UNFPA, otras agencias de las Naciones Unidas, y los organismos no gubernamentales, y reafirmar la llamada de la Declaración de Co. lombo sobre Población y Desarrollo para lograr una meta anual de un billón (mil millones) de dólares para la asistencia poblacional en el año 1984; y para los gobiernos donantes, dedicar una proporción significativa de su asistencia para el de. sarrollo a los programas de población. El incremento de asistencia internacional propuesto aquí requerirá que se fortalezca el rol y la función de la UNFPA.

23. Luchar por la interrupción de la migración masiva de refugiados causada por las agresiones políticas y militares, tomando nota especial de las resoluciones de las Naciones Unidas, al respecto.

24. Luchar por la suspensión de las "armas" como un paso hacia el desarme genuino y el aprovechamiento de los recursos que de esta suspensión se dispon. drían para dedicarlos a la solución de los problemas de población y desarrollo.

\section{Las Naciones Unidas:}

25. Convocar a una Conferencia Mundial de Población de Naciones Unidas en 1984, como fue recomendado en la Declaración de Colombo, que evaluaría el progreso de los últimos diez años desde la Conferencia de Bucarest sobre Pobla. ción, y haría propuestas para una acción futura.

26. Declarar un DIA DE POBLACION anual cuyos propósitos serían incre. mentar la conciencia y acrecentar la comprensión de los problemas de población tal como se relacionan con el desarrollo.

A las Naciones Unidas, a todas sus agencias y sus organismos, al Banco Mundial, a los bancos regionales de desarrollo y a las agencias intergubernamentales:

27. Aumentar el apoyo financiero y su contribución a los gobiernos de los países de Asia con el fin de sostener y promover la implementación de proyectos y programas y la integración de éstos con la población y el desarrollo.

28. Proseguir esta Conferencia de Parlamentarios Asiáticos sobre Población y Desarrollo con un apoyo para la organización de otras conferencias similares, al menos una vez cada tres años.

29. Mantener relaciones estrechas con grupos de Parlamentarios para la realización de esta labor y apoyar la organización de reuniones subregionales do Parlamentarios sobre el mismo tema con regularidad.

30. Apoyar los esfuerzos continuos de Parlamentarios a nivel asiático y subre. gional para coordinar de manera eficaz las actividades de los grupos nacionales de Parlamentarios para la Población y el Desarrollo.

31. Apoyar y fortalecer las instituciones existentes en los campos de salud re. productiva, tecnología de la anticoncepción, y estudios de la población y el desa. rrollo en Asia; establecer una mayor cooperación y colaboración entre los países asiáticos, y facilitar el desarrollo de personal capacitado y el avance de conocl. mientos en este campo que realmente acelerarían el proceso de integración de la población con el desarrollo.

32. Otorgarles una elevada importancia a los organismos no gubernamentales con el fin de transformar los programas de planificación familiar en un movi- 
miento amplio y popular, enfatizando el rol del sector privado para apoyar estos organismos.

33. Apoyar y consolidar los programas de los organismos no gubernamentales que conducen a la integración de los recursos, la población y el desarrollo, facilitando así el logro de metas nacionales. Con este fin, los organismos no gubernamentales (tales como la Federación Internacional de Planificación Familiar) (FIPF) deberían tener un rol clave y recibir más apoyo.

\section{Organismos no gubernamentales:}

34. Aumentar sus esfuerzos y su nivel de participación en la divulgación de información sobre temas de población y la implementación de programas de planificación familiar dentro del marco de las políticas nacionales, sobre todo en las áreas remotas e inaccesibles donde normalmente no se dispone de servicios de planificación familiar.

La prensa y otros medios de comunicacion:

35. Proveer una cobertura positiva de los temas de población y desarrollo para aumentar la percepción y la comprensión públicas de estos asuntos.

\section{Lideres religiosos:}

36. Tomar en cuenta el enorme impacto de las presiones de la población sobre el bienestar humano, espiritual y físico.

\section{Compromiso}

Nosotros los parlamentarios asistentes a esta Conferencia y de acuerdo con nuestras distintas capacidades como legisladores, líderes de la comunidad y representantes del pueblo, nos comprometemos y nos dedicamos a continuar y a iniciar y perseguir acciones dedicadas a lograr una integración eficaz de la población en las políticas y los programas de desarrollo.

\section{Como legisladores}

- Estimularemos el interés y crearemos conciencia y comprensión de las interrelaciones entre la población y el desarrollo entre nuestros hermanos parlamentarios.

- Promoveremos la formación de grupos de parlamentarios preocupados por la población y el desarrollo a nivel nacional, regional e internacional.

- Aseguraremos, a través de medidas legislativas apropiadas, que se provea una formación adecuada de recursos para la implementación de proyectos y programas diseñados para integrar la población con las políticas y programas de desarrollo.

- Participaremos y apoyaremos la implementación de políticas y programas con el objeto de facilitar la integración eficaz de la población con el desarrollo, y asegurar que su beneficio llegue a todos los niveles de la sociedad, sobre todo a los pobres y los menos privilegiados. 
- Aseguraremos que las discusiones y las deliberaciones sobre temas de población se guíen por el mejor interés de la nación en su totalidad.

\section{Como lideres de la comunidad}

- Generaremos el apoyo, la participación y el compromiso públicos para lograr el uso óptimo de recursos y la implementación de programas para mejorar el nivel socioeconómico del pueblo.

\section{Como representantes del pueblo}

- Nos dedicaremos a funcionar como un canal de comunicaciones para articular las necesidades y los problemas del pueblo, asegurar y participar en la evaluación de la implementación de políticas y programas, y luchar por lograr los ajustes y las mejoras necesarios.

- Nosotros, que somos el vínculo más íntimo entre el gobierno y el pueblo, nos dedicaremos a llevar el mensaje de las interrelaciones entre la población y el desarrollo a través de organismos populares.

\section{Finalmente $y$ en todos estos esfuerzos}

- Nos comprometemos a trabajar más íntimamente con otros organismos para realizar las metas y los objetivos de esta Declaración.

REPORTE DE LA REUNION-SEMINARIO SOBRE "PROPUESTAS INNOVADORAS EN LA ADMINISTRACION Y FINANCIAMIENTO DEL DESARROLLO URBANO" CELEBRADO EN SAN JOSE, COSTA RICA, DEL 23 AL 27 DE NOVIEMBRE DE 1981.

La discusión acerca de los ámbitos de competencia de los distintos niveles de gobierno, así como de las relaciones entre ellos (gobierno central-gobierno local), no ha sido desarrollada suficientemente en América Latina. Destacan contadas instituciones ${ }^{1} \mathrm{y} / 0$ estudios ${ }^{2}$ orientados a la administración de los gobiernos locales o regionales y muy pocas veces se han reunido ${ }^{3}$ los latinoamericanos interesados en estos temas.

Esto no quiere decir que no exista en nuestros países una tradición regionalista o localista tanto desde el punto de vista de la filosofía política ${ }^{4}$ como de los

1 Instituto Brasileiro de Administraçao Municipal (IBAM).

2 Centro Latinoamericano de Administración para el Desarrollo (CLAD). (1966)

3 BID-Reunión sobre Financiamiento Municipal en Latinoamérica, Washington

4 En Costa Rica, Brasil, México, entre otros. 
estudios urbano-regionales. ${ }^{5}$ Sin embargo, contrariamente a las tendencias manifestadas en algunos países desarrollados, en América Latina no parecen haber fructificado aún a niveles político, administrativo, cotidiano y práctico, estos intereses. Nuestros gobiernos municipales asi como las instancias de representatividad local urbana, se han dejado abandonados a su suerte y han perdido o bien nunca han conseguido realmente el status que, en algunos casos, formalmente se les reconoce. Su incapacidad para la autogestión, así como para generar recursos propios no se ha consolidado o se ha coartado en el proceso centralizador $y$ concentrador, tanto económico como político de nuestros países.

Los pasados 23-27 de noviembre de 1981, El Colegio de México (COLMEX) organizó bajo los auspicios del Banco Interamericano de Desarrollo (BID) una reunión-seminario en San José de Costa Rica sobre "Propuestas innovadoras en la administración y financiamiento del desarrollo urbano", contando con la colaboración local del Consejo Superior Universitario Centroamericano (CSUCA) y el Instituto Centroamericano de Administración Pública (ICAP).

La celebración de esa reunión constituía una de las actividades contempladas en el Convenio celebrado entre El Colegio de México y el BID para apoyar, durante el período de vigencia de dicho Convenio (1979-1982), la formación de profesionistas e investigadores en desarrollo urbano a nivel de maestría y en general las actividades docentes y de investigación de los problemas urbanos y regionales de los países latinoamericanos.

La agenda se estructuró alrededor de cuatro áreas temáticas, se presentaron 14 ponencias y se reservó una sesión de discusión general y conclusiones para el último día.

El programa fue el siguiente:

Tema I. Contexto sociopolitico de los gobiernos locales

1. "Notas sobre el poder local en el tercer mundo", José Ramón La. suén.

2. "Papel del municipio latinoamericano de la prestación y financiamiento de servicios públicos locales", Diogo Lordello de Mello.

Tema II. Consolidación del gobierno local

1. "Curitiba: Una experiencia en planeación urbana", Angel W. Bernal Acho.

2. "Municipalidad, asentamientos humanos y desarrollo urbano", Armando Araúz Aguilar.

3. "Capacitación y formación de cuadros en el nivel municipal: Experiencia reciente en México", José Antonio Aguilar Narvaes.

4. "Nueva ley de municipalidades en el Perú", Luis Bustamante Belaúnde.

Tema III. Administración urbana

1. "Oferta y demanda de servicios urbanos", Joao Ricardo Serrán.

2. "Un enfoque para el financiamiento de la vivienda", Jorge Carballo Wedel.

5 Por ejemplo: Lordello de Mello en L. Unikel y A. Necochea (comps.) (1975), así como varios trabajos en CLAD, publicados por SIAP (1976). 
3a. "La implantación de planes orientadores de inversiones urbanas: administración, financiamiento y recursos humanos. El caso de Matagalpa, Nicaragua", Mario Barahona Solís.

3b. "La descentralización administrativa: Participación y ejecución en los planes de desarrollo", Nelson Brown.

Tema IV. Financiamiento de obras y servicios municipales

1. "Consideraciones sobre el proceso de desarrollo urbano y su financiamiento", Jesús Rodríguez Montero.

2. "Crecimiento y reforma fiscal: Implicaciones recientes para la urbanización brasileña", Eduardo Sales Novaes.

3. "El problema financiero municipal en su evolución más reciente (1950-1979) y los determinantes estructurales de la crisis institucional del régimen municipal costarricense", Jose Luis Vega y Roderico Rodríguez.

4. "Financiación de proyectos de infraestructura urbana con relación de la capacidad de pago de los municipios y los beneficiarios", Juan Antonio Rodríguez Carazo.

Mesa Redonda: Innovaciones en la administración y el financiamiento del desarrollo urbano, coordinada por Eduardo Neira.

Respondiendo a la filosofía e intereses intelectuales del Ingeniero Luis Unikel (fundador del Area de Desarrollo Urbano y director del CEED, fallecido en enero de 1981), se invitó a participar a un grupo heterogéneo de personas constituido por académicos, políticos y planificadores para discutir desde sus particulares puntos de vista e intereses, los tópicos que se propusieron para la reunión. Los invitados provenían de instituciones académicas y centros de investigación, de instituciones financieras del sector público, de grupos de planificación urbana y regional, además de algunos alcaldes o sus representantes. Asistieron en total cuarenta participantes latinoamericanos y otros tantos observadores de instituciones locales (Costa Rica). ${ }^{6}$

Se intentaba con ello acercar los discursos de los planificadores, políticos y académicos con objeto de permitir, a unos, fundamentar teóricamente sus acciones y a los otros, aprender de la experiencia y la práctica y así, entre todos, reconstruir los posibles niveles explicativos del desarrollo urbano, local y sus vínculos con la política económica, nacional y regional, del gobierno central. Se buscaba, asimismo, abordar una temática no desarrollada suficientemente en los medios académicos y de investigación institucionales en América Latina; propiciar el inicio de trabajos de investigación alrededor de la compleja problemática que servía de tema a la reunión y finalmente, contribuir al fortalecimiento de las corrientes de pensamiento que apoyan el desarrollo de los niveles locales de gobierno, tradicionalmente tan castigadas en nuestros países.

\footnotetext{
${ }^{6}$ Se tuvo el honor de contar con la presencia del Sr. Presidente de la República de Costa Rica, Don Rodrigo Carazo Odio, quien declaró inaugurados los trabajos del seminario; asimismo, las palabras de Don Alvaro Saborío Ruiz, Ministro de Vivienda y Asentamientos Humanos de Costa Rica y del Lic. Carlos Arriola Woog, Secretario General de El Colegio de México, quien agradeció a los participantes su asistencia a dicha reunión.
} 
Sería pretensioso, sin embargo, pensar que todo ello se logró plenamente o que pudieran derivarse, en estos momentos, conclusiones finales. Pero no cabe duda de que la reunión permitió el intercambio de ideas y experiencias relevantes. No sólo se pudo aprender de experiencias pasadas, sino que de las discusiones pudieron rescatarse algunos elementos que permiten, nos parece, hacer planteamientos innovadores, reformular hasta cierto punto cuestiones que se han aceptado convencionalmente y juzgar acciones de gobiernos centrales o locales, algunas de las cuales han alcanzado la fase de implementación ${ }^{7}$ y otras solamente una fase de gestación.

En lo que sigue se destacan algunos de los tópicos que a nuestro parecer sobresalieron de las amplias discusiones desarrolladas a lo largo de la reunión.

Se señaló en primer lugar, que en algunos casos latinoamericanos puede apreciarse un movimiento desconcentrador reciente, no sólo a partir de la acción política deliberada, que aún es incipiente -sino más bien como resultado de consideraciones de tipo económico por parte de empresas y organizaciones tanto públicas como privadas. Esto se refleja en un patrón de comportamiento agregado de unidades económicas con efectos descentralizadores complejos que requieren de una respuesta por parte del Estado en todas sus implicaciones políticas, económicas y sociales en general. Es fácil ver en esto la necesidad de reforzar a los gobiernos locales (en particular, a las ciudades intermedias) y considerar seriamente la descentralización política y administrativa del sector público.

Para ello, sería necesario reflexionar sobre el origen y dinámica de nuestras poblaciones y sobre las formas de organización y evolución de los aparatos productivo y político de nuestras sociedades, con objeto de buscar las formas más adecuadas para la transformación paulatina del tradicional bloque monolítico del poder central y sus consecuencias concentradoras.

Hemos de pensar pues, en el proceso de descentralización, no sólo como una inquietud intelectual, sino como algo real, complejo y aparentemente contradictorio donde al tiempo que se predican e intentan medidas de reforzamiento y apoyo a los ayuntamientos, se les quita poder y funciones otorgándoselo a los organismos centrales.

Dentro de este juego, sin una clara concepción del proceso global de desarrollo en los países del área, será difícil calibrar las relaciones entre los poderes regional, local y central de forma que se favorezca efectivamente la democratización, trascendiendo las políticas de tipo paternalista y benefactor.

Es pues necesario de robustecer al poder local y tender a establecer una clara división de funciones y competencias entre poder central y local, para así sentar las bases de la articulación de distintas formas de transferencias reales de poder y de recursos.

Hubo, sin embargo, voces de cautela: si bien no todo es centralización y por lo tanto, no todo es, ni debe ser, descentralización, no puede aceptarse tampoco

7 Por ejemplo, "La Nueva Ley de Municipalidades del Perú" y la consolidación de corporaciones regionales en Ecuador constituidas por varios municipios, permitiéndoles mayor representatividad frente al gobierno central, así como soluciones a proyectos con problemas de economía de escala, etcétera. 
que una sea "mala" y la otra "buena". La dicotomía reduce la complejidad real de los procesos. ocultando las posibles soluciones. Estas no sólo deben ser innovadoras, sino - como alguno de los participantes señalaba- de sentido común. Por otra parte, los efectos, las soluciones no son homogéneas, no son finales. Habría que considerar para cada caso particular las necesidades y las posibilidades. En otras palabras. una cosa es el aspecto normativo (los deseos) y otra distinta la posibilidad de aprovechar los "espacios existentes" para poner en práctica y lograr realmente la descentralización, la autogestión, etcétera.

Descentralizar significa redistribución de poder, mayor participación en las decisiones que afectan a cada municipio, a cada barrio, a cada ciudad, pero también descentralización implica la posibilidad de generar recursos propios y poder disponer de ellos. Las propuestas innovadoras, por lo tanto, no estarían orientadas solamente a pedir más, sino en saber y poder emplear los recursos. Si bien, desde el punto de vista del gobierno central son pertinentes las preguntas sobre ¿qué financiamos; qué hacemos con los recursos; cómo responder y satisfacer los objetivos y necesidades legítimas de las mayorías?; sin duda que también se. ría importante preguntarse: ¿cómo funciona el sistema fiscal; es necesario un cambio? En resumen, para ser realistas habria que contestar al qué descentralizar y además al cómo y cuándo hacerlo.

En la búsqueda de los mecanismos internos de descentralización, no puede dejarse de lado la participación popular, la consulta previa. Parecería imposible hablar de descentralización sin implicar "democracia", es decir, participación, libertad de acción. Esto sin embargo, no debe quedarse en un nivel abstracto. La solución a las necesidades básicas es central en toda idea de democracia.

Respecto al cómo hacerlo, se dieron a conocer las más diversas experiencias:

- Acciones desde el poder central, tendientes a fortalecer las unidades menores como. por ejemplo, la creación de instituciones de crédito capaces de recibir apoyo financiero interno y externo para sus obras;

- Ejemplos de iniciativas y luchas generadas en los gobiernos locales, como los del Amazonas Ecuatoriano. que logran una injerencia importante en las decisiones centrales.

- Reformas legales y fiscales; creación de impuestos compartidos y establecimiento de convenios intergubernamentales como en Costa Rica, Perú, Nicaragua, México, etcétera.

- Formación de cuadros técnicos, al nivel de la planificación y la administración local, mediante cursos de capacitación, diseño de material didáctico y otros programas. Ejemplo de ello serían el IBAM en Brasil y la Dirección de

- Administración Urbana de la SAHOP en México.

- Apertura de sistemas de financiamiento de vivienda e infraestructura.

- Experiencias de planificación urbana, en muchos sentidos exitosas, como la de Curitiba en Brasil, etcétera.

Todos estos aspectos y algunos más que se expusieron a lo largo de las presentaciones de los ponentes y sus comentaristas dejaron ver la necesidad de estudiar, investigar, medir, cuantificar, explicar la problemática alrededor de la administración y financiamiento del desarrollo urbano, así como de los procesos que segu- 
ramente se estimularán o reforzarán una vez que se pase de la mera retórica del discurso político a la acción descentralizadora.

La lógica de la centralización como señalaba un ponente, no parece, al menos en nuestros paises haberse roto. En este sentido la evaluación de las acciones particulares y el comportamiento general de las instituciones financieras del sector público orientados al financiamiento del desarrollo urbano y regional tendría que responder la pregunta de ¿por qué, si realmente existen los deseos de resol. ver problemas y ofrecer soluciones no ha habido resultados satisfactorios, será que no existen posibilidades reales de solución vía la aplicación de instrumentos (financieros) convencionales?

Otro señalamiento importante con implicaciones metodológicas, pero sobre todo políticas, se refería a que los movimientos regionalistas muchas veces no son progresistas, sino sólo anticentrales, mientras que por el contrario los centralistas en ocasiones paradójicamente son progresistas. En ocasiones también el esfuerzo descentralizador cuando se gesta desde el centro, tiende a ser concentrador: las autoridades locales se designan y controlan desde el centro. Esto, ya sea por cuestiones políticas y/o económicas que responden a intereses sectoriales o de grupos o fracciones sociales, ha mantenido en su histórica debilidad al municipio, al gobierno local que no teniendo representatividad y estando comprometido con el poder central, se ve reducido a una posición pasiva frente a las necesidades e intereses locales.

$\mathrm{Si}$ acaso pudieran ser resumidas las consideraciones hechas y las opiniones expresadas durante una semana de intensa participación por parte de los asistentes a la reunión, podría decirse lo siguiente:

a) Es importante revertir el orden de prioridades en el financiamiento del desarrollo urbano... (tanto en el interior de las grandes ciudades o regiones metropolitanas como dentro de la jerarquía urbana en favor de los municipios y localidades medianas y pequeñas).

b) Es necesario privilegiar aquellos instrumentos y mecanismos participativos y democráticos, sobre los excluyentes y autoritarios... (habría que distinguir, como señalaba un ponente, entre "Estado Democrático" y sociedad democrática).

c) Se torna urgente convencernos y probar las posibilidades de gestión y competencia que los niveles locales del gobierno tienen para llevar a cabo funciones más allá de las mínimas tradicionales de prestar servicios públicos locales. (Es de notar sin embargo, que en la mayoría de los casos el gasto corriente de la alcaldía absorbe, si no todos, una cada vez mayor proporción de los recursos existentes o a su alcance).

d) Habría que dejar de hablar de lo formal, lo normativo y lo simbólico y pasar a haubiar de lo real, o en otras palabras, desmitificar el tratamiento de los problemas y de los mecanismos que condicionan los aspectos sociales, económicos, políticos y culturales de las sociedades latinoamericanas, pasando al estudio profundo de los aspectos específicos y puntuales que lleven a soluciones concretas y cambios, aunque sea graduales, pero reales de la situación social de las comunidades locales en nuestros países.

Más que de conclusiones se trata de inquietudes. $Y$ si hubiese que sacar una conclusión, quizá la más importante sería que al término de la reunión los mar- 
cos teóricos propuestos empezaron a llenarse de contenido y a replantearse a la luz del intercambio de experiencias concretas.

Dada la complejidad de los temas, en esta reseña se señalaron sólo algunas de las cuestiones tratadas en la reunión. Se espera sin embargo, que puedan servir para generar una serie de preguntas que habrá que contestar en el futuro inmediato, si se quiere responder con prontitud y adecuadamente a las exigencias actuales de nuestros países, en lo que respecta al financiamiento y administración del desarrollo de sus ciudades y la participación efectiva de sus comunidades regionales y locales.

Boris Graizbord María Eugenia Negrete Crescencio Ruiz Ch. 